\title{
A Novel Approach to Identify Wood Species Optically using Fluorescence Lifetime Imaging Microscopy
}

\author{
Nina Leiter ${ }^{1}$, Maximilian Wohlschläger ${ }^{1}$, Veronika Auer ${ }^{1}$, Martin Versen ${ }^{1}$, Christian Laforsch ${ }^{2}$ \\ 1 Technical University of Applied Sciences Rosenheim, Hochschulstr. 1, 83024 Rosenheim, Germany \\ 2 University Bayreuth, Universitätsstr. 30, 95447 Bayreuth, Germany \\ martin.versen@th-rosenheim.de
}

\begin{abstract}
Summary:
This contribution presents the results obtained with fluorescence lifetime imaging microscopy (FLIM) within the optical identification and differentiation of the four wood species walnut, beech, spruce, and maple. The experimental setup as well as the evaluation algorithm, with which the experiments were carried out, is explained briefly.
\end{abstract}

Keywords: Fluorescence Lifetime, wood species identification, Fluorescence, wood, FLIM

\begin{abstract}
Introduction
Wood is the world's most important renewable raw material, which can be used several times within a life cycle, as material and for energy production at the end of the life cycle. The areas of material application are manifold and span from building, construction, engineered material and interior products. After the specific product life time, the then so called post-consumer wood can be recycled and reprocessed. Actually, recycled wood is mainly used within particle boards (up to $33 \%$ [1]). However, this material flow represents only $17 \%$ of the yearly resulting post-consumer wood in 2016. If the $6 \%$ of polluted wood is taken into account, approximately $77 \%$ of the post-consumer wood would potentially be available [2]. Therefore, the noninvasive and in-situ identification of wood species opens new material utilisation pathways of post-consumer wood, e.g. in massive wood products or in biorefineries.
\end{abstract}

At the moment, there are two main techniques under research to identify wood: near-infrared spectroscopy (NIRS) and X-ray fluorescence spectroscopy (XRF). According to [3], it is possible to identify wood types using NIRS. Unfortunately, the detection is complicated because the NIR spectrum is superimposed with the absorption band of the variable moisture content in wood. The second method to identify wood is XRF [4]. This method is not appropriate for an automated wood identification, since only pollutants such as heavy metals with high atomic numbers can be identified.. Thus, there is a demand for the development of a reliable and automatable method of wood identification and sorting.
The main component responsible for fluorescence signal of wood under excitation in the ultraviolet (UV) of visual (VIS) region is lignin in the cell walls [5]. The spectral fluorescent properties can be used for an identification purpose of wood, but vary in a significant manner [6]. Also, the fluorescence lifetime, measured in time-domain, can be used to identify wood species $[7,8]$. In order to use the method of fluorescence decay time measurement in the time domain, fast synchronization and expensive equipment is required, which seems uneconomical.

Alternatively, the fluorescence decay time can be measured in the frequency domain with a FD-FLIM (frequency domain fluorescence lifetime imaging microscopy) camera. In this case, larger areas can be examined at once and the costs are considered to be lower. For this reason, the FD-FLIM method is investigated in this contribution.

\section{Description of the New Method}

In the FD FLIM method, the sample is excited by a sinusoidal or rectangular modulated light signal. The resulting fluorescence signal (emission) is phase shifted to the excitation signal. If the phase shift $\phi$ is measured at a known modulation frequency $\omega$, the fluorescence lifetime $\tau$ can be calculated according to eq. (1).

$$
\tau=\frac{1}{\omega} \cdot \tan (\phi)(1)
$$

The PCO AG from Kelheim has developed an FD-FLIM camera system, which can measure fluorescence decay times from 100 ps up to $100 \mu s$ with a dynamic range of 10 bit [9]. For 
each of the $1008 \times 1008$ pixels, the intensity, the phase shift, the modulation depth and the phase and modulation lifetime can be determined.

The experimental setup consists of a laser diode Omicron PhoxX-488 that emits a sinusoidal modulated light signal of $488 \mathrm{~nm}$ wavelength with a definite modulation frequency $\omega$. As fluorescence detector a pco.flim camera is used. Two optical filters placed in the excitation and emission paths narrow the bandwidth and block stray and reflected light. All components are assembled to a Motic PSM 1000 microscope, which contains a $20 x$ magnification objective.

Four images of each sample at random positions are captured with the experimental setup and evaluated with a Gaussian analyzation as described in [10].

For the experiments wooden probes of walnut, beech, spruce and maple with a size of $2 \times 2 \times 0.5$ $\mathrm{cm}$ are prepared.

\section{Results}

The measured fluorescence decay times for each wood specie are presented in Fig. 1. The measured values fit very well to Gaussian distributions. With this representation, a graphical differentiation and identification of the individual types of wood is possible.

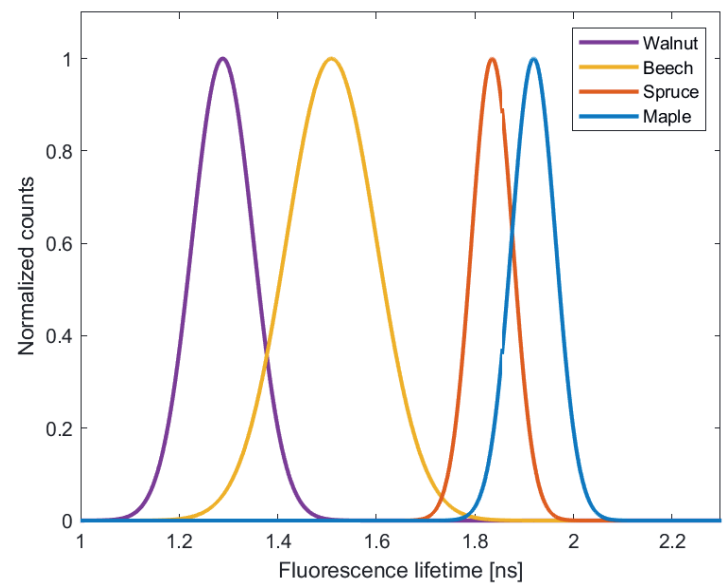

Fig. 1. Visualization of the measured fluorescence lifetimes of the four different wood species.

In addition, the calculated mean values of the fluorescence lifetimes and the corresponding standard deviations show similar results assuming a Gaussian distribution (see Tab. 1). Thus, an identification is also possible by calculation.

In summary, the wood species are unambiguously distinguishable and identifiable graphically and by calculation due to their characteristic fluorescence lifetime. This study clearly reveals the potential of the FD-FLIM technique for wood identification procedures. In the future, addi- tional wood species should be investigated to exploit the full potential.

Tab. 1: Calculated mean values of the fluorescence lifetime and corresponding standard deviation.

\begin{tabular}{|c|c|}
\hline Wood species & Fluorescence lifetime [ns] \\
\hline Walnut & $1.29 \pm 0.06$ \\
\hline Beech & $1.51 \pm 0.09$ \\
\hline Spruce & $1.84 \pm 0.04$ \\
\hline Maple & $1.92 \pm 0.04$ \\
\hline
\end{tabular}

\section{References}

[1] Klinker G., In praise of recycled wood. PFL Inspirations, 2016

http://inspirations.pfleiderer.com/13680-in-praiseof-recycled-wood. (Last Accessed 23 Nov. 2020)

[2] Flamme S., Hams S., Bischoff J., Fricke C., Evaluierung der Altholzverordnung im Hinblick auf eine notwendige Novellierung, Umweltbundesamt, Münster, 2019, ISSN 1862-4804

[3] Mauruschat, D., Plinke, B., Aderhold, J., Gunschera, J., Meinlschmidt, P., Salthammer, T., Application of near-infrared spectroscopy for the fast detection and sorting of wood-plastic composites and waste wood treated with wood preservatives. Wood Sci Technol 50, 2016, pp. 313-331. DOI: 10.1007/s00226-015-0785-x

[4] Hasan R., Solo-Gabriele, H.; Townsend T., Online sorting of recovered wood waste by automated XRF-technology: part II. Sorting efficiencies. In: Waste management, 31, 4, 2011a, pp. 695-704. DOI: 10.1016/j.wasman.2010.10.024.

[5] Albinsson, B., Li, S., Lundquist, K. \& Stomberg, R. (1999) The origin of lignin fluorescence. J. Mol. Struct. 508, 19-27.

[6] Donaldson L.A., Softwood and hardwood lignin fluorescence spectra of wood cell walls in different mounting media. IAWA J 34, 2013, pp. 3-19. DOI: $10.1163 / 22941932-00000002$

[7] Donaldson L.A., Radotic K, Fluorescence lifetime imaging of lignin autofluorescence in normal and compression wood. J Microsc., 251, 2, 2013, pp. 178-187, DOI: 10.1111/jmi.12059

[8] Burkhardt, H. Die Fluoreszenzlebensdauer in der Holztechnologie. Holztechnologie 60,6 , 2019, pp. 26-30.

[9] Chen H., Holst G., Gratton E., Modulated CMOS Camera for Fluorescence Lifetime Microscopy, Microscopy Research \& Technique, 78, 12, 2015, pp. 1075-1081. DOI: 10.1002/jemt.22587

[10] M. Wohlschläger, G. Holst, M. Versen, A novel approach to optically distinguish plastics based on fluorescence lifetime measurements, IEEE Sensor Applications Symposium 2020, Kuala Lumpur, Malaysia, 2020, pp. 1-6. DOI: 10.1109/SAS48726.2020.9220084 